Research Paper

\title{
Chloroquine Promotes the Anticancer Effect of TACE in a Rabbit VX2 Liver Tumor Model
}

Lu Gao $^{1 *}$, Jian-rui Song1 ${ }^{*}$, Jian-wei Zhang1 ${ }^{*}$, Xue Zhao ${ }^{1,2}$, Qiu-dong Zhao1, Kai Sun1,2, Wei-jie Deng1, Rong $\mathrm{Li}^{1}$, Gang Lv ${ }^{1}$, Hong-yan Cheng ${ }^{1}$, Li-xin Wei ${ }^{1,2}$

1. Tumor Immunology and Gene Therapy Center, Eastern Hepatobiliary Surgery Hospital, The Second Military Medical University, Shanghai, China;

2. Central laboratory, renji hospital, Shanghai Jiaotong University School of Medicine, Shanghai, China.

* These authors contributed equally to this work.

$\triangle$ Corresponding author: Lixin Wei, MD., Ph.D. Tumor Immunology and Gene Therapy Center, Eastern Hepatobiliary Surgery Hospital, The Second Military Medical University, 225 Changhai Road, Shanghai 200438, China. Tel: +86-21-81875331 Fax : +86-21-65566349 E-mail: lixinwei@smmu.edu.cn.

() Ivyspring International Publisher. This is an open-access article distributed under the terms of the Creative Commons License (http://creativecommons.org/ licenses/by-nc-nd/3.0/). Reproduction is permitted for personal, noncommercial use, provided that the article is in whole, unmodified, and properly cited.

Received: 2013.0I.2I; Accepted: 2013.03.19; Published: 2013.03.28

\begin{abstract}
Background: To investigate the efficacy of TACE combined with CQ, an autophagic inhibitor, in a rabbit VX2 liver tumor model.

Methods: Tumor size was measured. And tumor growth rate was calculated to examine the effect of the combined treatment. Apoptosis was detected by TUNEL assay. Meanwhile, autophagic activity was detected by immunohistochemistry and Western blotting to investigate the mechanism underlying. Liver function was also examined to assess feasibility and safety of the combined therapy.

Results: Tumors in the control grew more than 4 times bigger after 14 days, while that in the group of TACE alone just showed mild growth. But a slight shrinkage was shown after the treatment of CQ+TACE. Growth ratio of TACE alone was $96.45 \% \pm 28.958 \%$ while that of CQ+TACE was $-28.73 \% \pm 12.265 \%$. Compared with TACE alone, necrosis in CQ+TACE showed no significant difference, however, the apoptosis was much higher. There were only $14.8 \pm 3.11 \%$ apoptotic cells in TACE, but $33 \pm 4.18 \%$ in CQ+TACE, which suggests the increased apoptosis in CQ+TACE contributed to the decrease of tumor volume. In terms of autophagic activity, the result is negative when we immunostained sections of the control with LC3 antibody, but positive in TACE alone and CQ+TACE. And the result of Western blot showed that there was just a low level of LC3 II expressed in the control and CQ alone, but higher in TACE, and much higher in CQ+TACE because CQ inhibited its degradation in autophagy. Compared with control, p62 decreased in TACE, but the decrease was partially reversed in CQ+TACE. In addition, toxicity of CQ+TACE was assessed not higher than TACE alone, which supports the safety of CQ+TACE.

Conclusion: $C Q+T A C E$ works better than TACE alone in rabbit VX2 liver tumor model because CQ inhibits autophagy induced by TACE. The inhibited autophagy loses its resistance to apoptosis that apoptosis increased, which contributes to the inhibition of tumor growth. This study indicates $\mathrm{CQ}$ may be a promising adjuvant to promote the effect of TACE.
\end{abstract}

Key words: Hepatocellular carcinoma, transcatheter arterial chemoembolization, chloroquine, apoptosis, autophagy.

\section{Introduction}

Hepatocellular carcinoma (HCC) ranks the fifth most common cancer and is the third leading cause of death from cancer worldwide [1]. Although surgery is the optimal treatment for liver cancer, only 10\%-30\% of patients are able to take the radical resection as most of them are detected at advanced stages when 
they have lost the opportunity of surgical therapy [2, 3]. For those unresectable HCC, transcatheter arterial chemoembolization (TACE) is the most widely used interventional procedure [4-6]. In addition, TACE has been used as a preoperative or postoperative adjuvant therapy in patients with respectable HCC in attempt to improve survival $[7,8]$. Therefore, about $95 \%$ of patients with primary liver cancer require TACE treatment.

However, the effect of TACE is unsatisfactory. It is reported that preoperative TACE (injection of chemotherapeutic agents mixed with lipiodol into the hepatic artery supplying the tumor, followed by the administration of embolizing agents such as Gelfoam particles) cannot reduce the recurrence or prolong survival after curative resection of HCC [7, 9], and that TACE appears to be no better than transarterial embolization (TAE, the embolization of hepatic artery without using any chemotherapeutic agents) [10, 11] .The fact that TACE is reported to be no better than TAE, implies that the effect of chemotherapeutic agents in TACE is uncertain. Hypoxia, resulted from embolization, is probably one of the most important factors rendering chemoresistance of cancer cells after TACE $[10,12]$.

Our previous study found that hypoxia mediates the chemoresistance of hepatocellular carcinoma cells by inducing autophagy [13]. Autophagy enhances the survival of hepatocellular carcinoma cells in hypoxia and then decreases their apoptotic potential, and accordingly the cells become resistant to chemotherapeutic agents. Thus combining autophagic inhibitor with chemotherapeutic agent may promote the apoptosis of hepatocellualar carcinoma cells in hypoxia, which in turn may promote the efficacy of TACE. One of the widely used autophagic inhibitors is chloroquine (CQ) which is a traditional drug for treatment of malaria [14].In recent years; CQ is specially used as an autophagic inhibitor. It is a weak base and usually trapped in acidic cellular compartments, such as lysosomes [15]. The deacidification of lysosomes by CQ impairs the activity of most lysosomal proteases that it inhibits the last step of autophagic degradation process, and consequently inhibits autophagy [16].

Autophagy is a catabolic process that enables cells to recycle amino acids and other intracellular nutrients, and to obtain energy from recycled materials [17]. The autophagy process occurs in three steps: (1) autophagosome formation; (2) lysosomal fusion with the autophagosome; and (3) lysosomal degradation [18]. As for the measurement of autophagy in vivo, immunodetection of LC3 in tissue sections is worth noting [19]. LC3, a marker of autophagosomes in mammalian cells, is activated and relocalized to intracellular vesicles when the lipid bilayer structure sequesters cytoplasm to form autophagosomes [20]. In addition, p62 is a multifunctional protein that binds to LC3 and to ubiquitinated proteins, which mediates the recognition of protein aggregates for autophagic clearance [21, 22]. The accumulation of p62 marks the dysfunctional autophagy which is not enough to process the damaged proteins bound to p62 [23-25].

The objectives of this study were 1) to examine the efficacy of the combination of TACE and CQ, an autophagic inhibitor, in a rabbit VX2 liver tumor model; 2) to explore the possible mechanism in vivo and 3) to evaluate the safety and clinical feasibility of this combination. To test the hypothesis that the combination of autophagic inhibitor and TACE may enhance the efficacy of TACE, we added autophagic inhibitor-CQ into the mixture of lipiodol and chemotherapeutic agent, then measured the tumor size and calculated the tumor growth inhibition ratio to examine the effect of combined treatment. We also detected the apoptosis and autophagic activity to validate the underlying mechanism. Finally, liver function was tested to explore the feasibility and safety of the therapy.

\section{Materials and methods}

All experimental procedures have been approved by the Institutional Animal Care and Use Committee of Second Military Medical University (Shanghai, China).

\section{Establishment of the animal model}

Adult New Zealand White rabbits, weighing 2-2.5kg, provided by the Experimental Animal Center of Second Military Medical University were used. Tumor implantation was performed according to the method outlined by Liang et al [26]. Briefly, solid tumors for implantation were obtained by injecting VX2 tumor cell suspension into both thigh muscles of a carrier rabbit. Two weeks later, the solid tumors were harvested from the carrier rabbit. Then a rabbit VX2 liver tumor model was induced by inserting 2-3 pieces of approximately $1 \mathrm{~mm}^{3}$ harvested tumors into one location in the left lobe of the liver of the rabbits for experiments. Fourteen days after tumor implantation, when the tumors were examined to be $15-30 \mathrm{~mm}$ in diameter on the CT scan, the rabbits were used for experiments.

\section{Experimental design}

Forty rabbits with VX2 tumors transplanted to their livers were divided into four groups with ten rabbits each for the study. The location and size of the tumor were measured with CT scan. The tumor volume $(\mathrm{V})$ was calculated according to the following 
equation $[27,28]: \mathrm{V}=\mathrm{a} \times \mathrm{b}^{2} / 2$, where $\mathrm{a}$ is the longest diameter and $b$ is the shortest diameter of the tumor. Depending on the calculated tumor volume, rabbits with similar-size tumors were distributed evenly to each group. Twenty rabbits were administered with TACE with the solution: TACE alone, $2 \mathrm{mg} / \mathrm{kg}$ cisplatin and $0.2 \mathrm{ml} / \mathrm{kg}$ lipiodol $(\mathrm{n}=10)$; $\mathrm{CQ}+\mathrm{TACE}, 2$ $\mathrm{mg} / \mathrm{kg}$ cisplatin and $60 \mathrm{mg} / \mathrm{kg}$ CQ mixed into 0.2 $\mathrm{ml} / \mathrm{kg}$ lipiodol $(\mathrm{n}=10)$. As a CQ alone group (CQ), rabbits were treated with $60 \mathrm{mg} / \mathrm{kg}$ CQ dissolved in $0.2 \mathrm{ml} / \mathrm{kg}$ physiological saline $(\mathrm{n}=10)$. And in the control group $(\mathrm{CON})$, ten rabbits were treated with 0.2 $\mathrm{ml} / \mathrm{kg}$ physiological saline.

\section{TACE}

Two weeks after implantation, TACE was administered. General anesthesia was induced and an incision was made to expose the right common femoral artery. When it was adequate, A 4-F vascular was inserted as a substitute for an arterial sheath (Terumo, Tokyo, Japan).With 4-F Cobra visceral catheter (Terumo), celiac angiography was performed to identify the feeding arteries and surrounding vascular anatomy. Then, the 3-F catheter system(Terumo) was advanced super selectively into the left hepatic.

After positioning the catheter within the left hepatic artery, the indicated substance or mixture was injected. For the TACE and CQ+TACE group, a mixture of cisplatin $(2 \mathrm{mg} / \mathrm{kg})$ and Lipiodol $(0.2 \mathrm{ml} / \mathrm{kg})$ was injected carefully, followed by the embolization of the gelatin sponge particles (Lipiodol Ultra-Fluide, Labo-ratoire guerbet, Aulnay-Sous-Bois, France) until flow reduction was observed. After the embolic material was injected the catheter and sheath was removed and the common femoral artery was ligated.

\section{Antitumor effect assay}

Follow-up CT examination was performed 1 and 14 days after the treatments to identify deposits of lipiodol and to measure the tumor volume and tumor growth inhibition ratio. The tumor volume was measured and the tumor growth ratio was calculated by comparing the tumor volume obtained before $(\mathrm{Vb})$ and 14 days after the treatments $(\mathrm{Va})$ with the formula: $(\mathrm{Va} / \mathrm{Vb}-1) \times 100$.

\section{Histological and Immunohistochemical Ex- amination}

At the 14th day after the treatments, the rabbits were sacrificed and the intact livers were removed. Formaldehyde-fixed, paraffin-embedded tissue blocks were prepared from tumor tissues and cut into $4 \mu \mathrm{m}$ thick sections. For hematoxylin and eosin staining (H\&E), the sections were stained by hematoxylin and eosin and evaluated with a light microscope. And for the immunohistochemical examination, all paraffin embedded sections were dewaxed in xylene and rehydrated in graded ethanol solutions. To block the endogenous peroxidase, the slides were treated with $3 \% \mathrm{H}_{2} \mathrm{O}_{2}$ for $20 \mathrm{~min}$, then washed with $\mathrm{ddH}_{2} \mathrm{O}$ three times (5 min each). Antigens were retrieved with sodium citrate buffer (0.01M citric acid: $\mathrm{pH}$ 6.0) for 10 min at $100^{\circ} \mathrm{C}$ in boiling. Next, sections were incubated with $10 \%$ BSA for $30 \mathrm{~min}$ at room temperature to reduce nonspecific binding, and then the primary antibodies (anti-HIF-1a, anti-Bax and anti-Bcl-2 from Santa Cruz Biotechnology, CA, USA;anti-LC3 and anti-p62 from Novus Biological, Littleton, CO)were applied to the sections at $4^{\circ} \mathrm{C}$ overnight. After washing in PBS three times (5 min each), sections were incubated with anti-mouse or anti-rabbit IgG peroxidase conjugated secondary antibodies (Bioworlde Technology) at $37^{\circ} \mathrm{C}$ for $30 \mathrm{~min}$. Bound secondary antibody was detected by the 3,3'-diaminobenzidine (DAKO) chromogen by standard methods.

\section{Evaluation of apoptosis}

The terminal deoxynucleotidyltransferasemediated nick-end labeling (TUNEL) assay was performed according to the instructions in the reagent kits (Roche Molecular Biochemicals). The number of apoptotic bodies was determined and expressed relative to the total number of cells within 10 random fields $(\times 200)$ of cells. In each field, the number of positive cell nuclei was calculated among 100 liver cells nuclei. The rate of apoptotic cell nuclei is defined as apoptotic positive cell nuclei/total cell nuclei in the field.

\section{Measurement of liver function}

Blood samples were collected from all rabbits before and at the 3rd day, the 7th day, and the 14th day postoperatively. The plasma alanine aminotransferase (ALT) and aspartate aminotransferase (AST) levels were tested with a biochemical autoanalyzer (Fuji Medical System, Tokyo, Japan) according to the manufacturer's instructions.

\section{Western Blotting}

Before immunoblotting, cells were lysed in RIPA lysis buffer (Beyotime) with $1 \mathrm{mM}$ PMSF and the protein concentrations were determined with a BCA detection kit (Thermo). An equal amount of protein from each sample was separated by sodium dodecyl sulfate-polyacrylamide gel electrophoresis (SDS-PAGE), transferred onto a NC membrane. After blocking with $5 \%$ non-fat milk, the protein on membrane was incubated with specific primary antibody against LC3(Novus Biologicals, Inc) and $\beta$ - actin (Santa Cruz), followed by incubation with a second 
antibody. Immunoreactivity was detected by the BeyoECL Plus substrate system (Beyotime).

\section{Statistical analysis}

Quantitative data were expressed as mean \pm SD. Significance between groups was performed with the Kruskal-Wallis test and Mann-Whitney U test. $P<0.05$ was considered statistically significant. Statistical analysis was performed with GraphPad Prism 5.0 software.

\section{Results}

\section{Efficacy of CQ plus TACE is better than TACE}

In the previous study, it is found that autophagy mediates the chemoresistance of hepatocellular carcinoma cells in hypoxia [13]. Then we tried to combine autophagic inhibitor-CQ with TACE to investigate whether the addition of CQ could increase cell death by sensitizing cancer cells to chemodrug in the hypoxic microenvironment caused by embolization, which was done in a rabbit VX2 liver tumor model. CQ was added into the mixture of cisplatin and lipiodol, then all of them were injected into the hepatic artery by connecting tube, followed by perfusion of embolizing agent-gelfoam. To detect whether hypoxia was induced by TACE, the major transcription factor HIF-1 that is specifically activated during hypoxia[29, 30] was examined by immunohistochemistry. Sections were stained with HIF-1a antibody because within the two subunits of HIF-1: HIF-1a and HIF-1 $\beta$, HIF-1a is the only hypoxia dependent one. Fig.1A showed that HIF-1a was significantly upregulated by TACE, which indicates the typical hypoxic environment resulted from TACE. In addition to TACE and CQ+TACE, results in the control and $\mathrm{CQ}$ alone were also positive, which may contribute to the hypoxia existed in solid tumors. Tumor volumes were examined 14 days after TACE. On contrary to similar sizes of all tumors before treatments, the sizes at the 14th day were significantly different from one another. The average size of tumors in the control was $21.146 \pm 1.779 \mathrm{~cm}^{3}$, while that in CQ alone and TACE group was respectively 17.997 $\pm 2.541 \mathrm{~cm}^{3}$ and $7.858 \pm 1.158 \mathrm{~cm}^{3}$. But it was only $3.646 \pm 1.317 \mathrm{~cm}^{3}$ in the CQ+TACE group (Fig.1B). According to the result of growth rate, tumor in CQ+TACE showed a slight shrinkage, and mild growth was shown in TACE alone, and both tumors in CQ alone and the control grew markedly (Fig.1C). Growth rate in CQ+TACE was $-28.73 \% \pm 12.265 \%$, while that in TACE was $96.45 \% \pm 28.958 \%$. They were significantly different with each other $(p=0.001)$, which indicates the enhanced antitumor effect of CQ+TACE, although both were lower than that in the control $(428.65 \% \pm 44.472 \%)$. What should be noted is that growth rate in CQ alone was $349.925 \% \pm 63.525 \%$, which was not statistically different from control, indicating CQ alone have no anticancer effect, at least at the concentration used in this study. In conclusion, CQ enhances the efficacy of TACE in the rabbit VX2 liver tumor model.
A

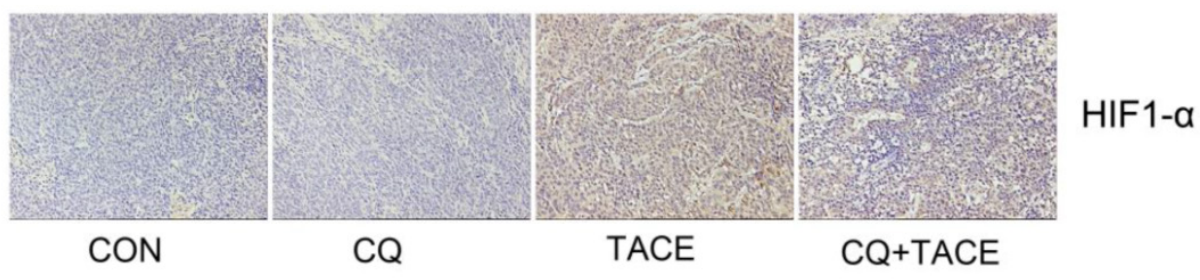

\section{A}

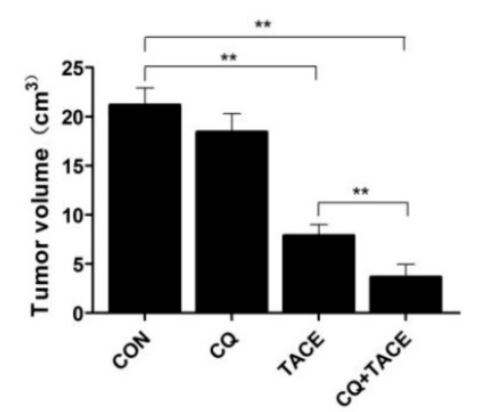

B

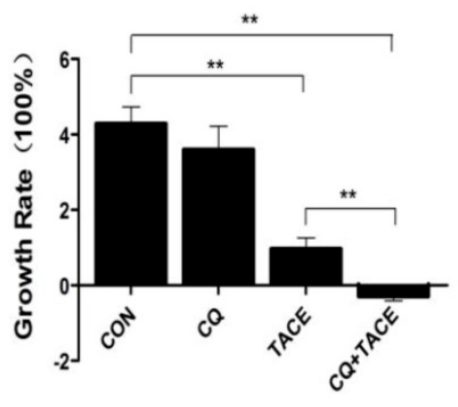

C

Fig I. Chloroquine (CQ) enhances the antitumor effect of TACE in vivo. (A) Expression levels of HIF-I $\alpha$ in each group I4 days after the treatment. A marked positive area was observed in the group of TACE and CQ plus TACE ,compared with those in control and CQ alone group. (B) Fourteen days after treatments indicated, the rabbits were sacrificed and tumor sizes were measured. Tumor volumes after the treatment of TACE and CQ+TACE were significantly smaller than that of the control. And compared with the tumor volume in TACE group, the volume in CQ+TACE was much smaller. (C) The growth ratio was calculated according to the tumor volumes gained. In CQ+TACE group, compared with the initial tumor volume, the volume 14 days after treatment shrank minimally. The tumor volume of rabbits received TACE alone grew mildly. On the contrary, the volumes in $\mathrm{CQ}$ alone group and the control increased markedly. The values are shown as means \pm SD. Statistically significant differences are marked $* * *<0.0 \mathrm{I}$. 


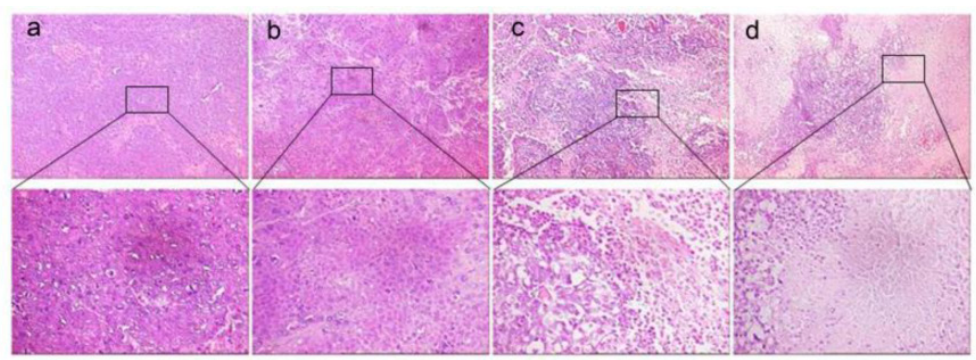

H\&E

A

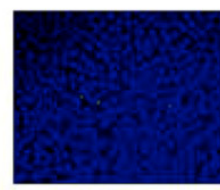

CON

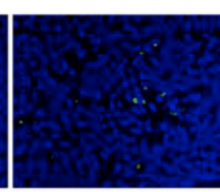

$\mathrm{CQ}$

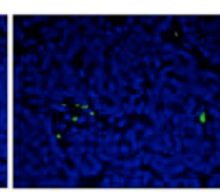

TACE

B

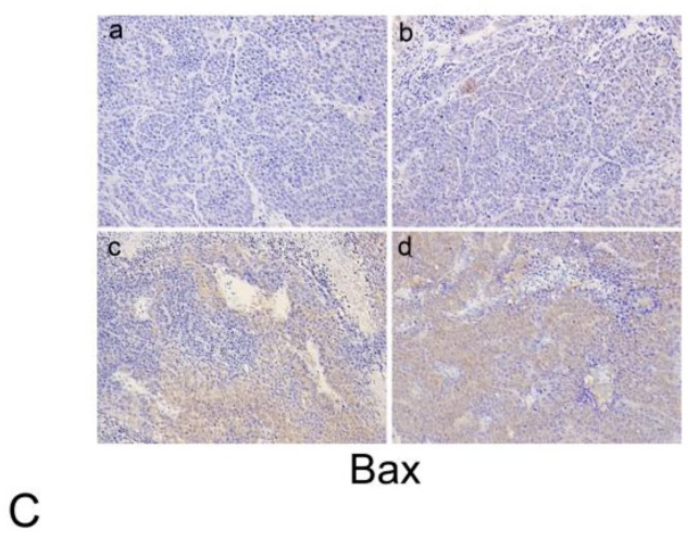

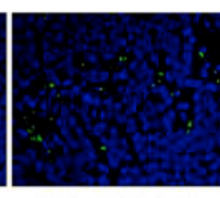

CQ+TACE
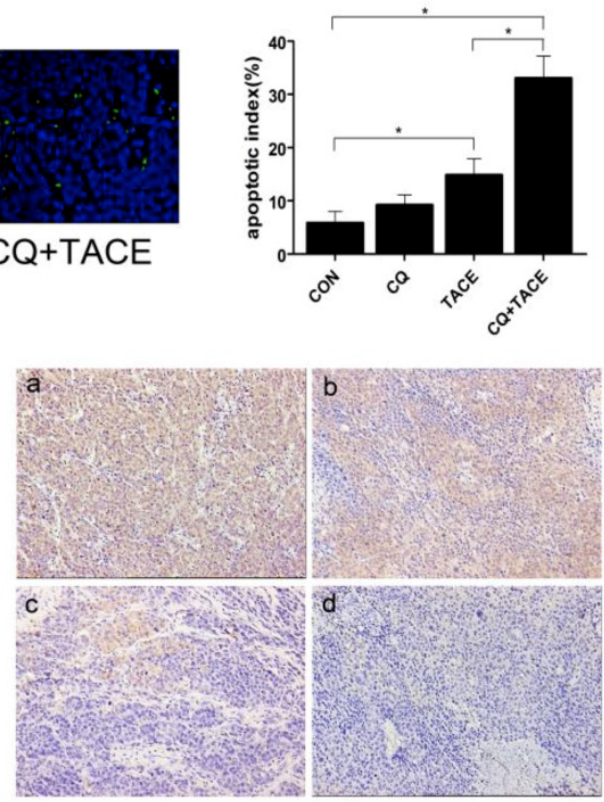

Bcl-2

Fig 2. Apoptosis increases when combine CQ with TACE. (A) The histological sections of VX2 liver tumors I4 days after treatments were stained with hematoxylin and eosin (H\&E). The representative images were shown with original magnification of $\times 200$ and $\times 400$ (inset). Viable tumor cells were found in tumor tissues of the control (a) and CQ alone (b) group. However, tissues after the administration of TACE (c) or CQ+TACE (d) underwent massive necrosis. (B) Apoptosis detection in different tumor tissues I4 days after the treatments by TUNEL. Representative images were shown $(\times 200$, left panel). Scattered positive cells were found in the group of TACE alone, while nearly none was found in the control and CQ alone group. But a significant increase was observed in the CQ+TACE. Quantitative analysis of TUNEL positive cells was on the right panel. Data (means \pm SD) represent the means of three independent experiments. ${ }^{*} p<0.05$. (C and D) The expression of Bax (C) and Bcl-2 (D) was analyzed by immunohistochemistry $(\times 200)$. Two weeks after the treatment, the VX2 liver tumor tissues in each group were collected and prepared for immunohistochemistry with $\mathrm{Bax}$ and $\mathrm{Bcl}-2$ antibodies. It is evident that there are larger amount of Bax-positive and less Bcl-2-positive cells in CQ+TACE group (d) than the TACE alone (c). And little Bax-positive and abundance of Bcl-2-positive ones are shown in the control (a) and CQ alone (b) groups.

\section{Apoptosis increases in CQ plus TACE}

TACE inhibits the tumor growth through necrosis resulted from embolization and apoptosis caused by targeted chemotherapy [31, 32]. CQ enhances the antitumor effect of TACE, but it is got by increase in necrosis or apoptosis or both is not clear. We examined necrosis and apoptosis by H\&E and TUNEL staining respectively. As shown in Fig.2A, both treatments of TACE and CQ+TACE caused obvious necrosis in tumor; however, there was no significant difference between them. On the contrary, apoptosis in CQ+TACE was significantly higher than that of TACE alone (Fig.2B). There were $14.8 \pm 3.11 \%$ apop- totic cells in TACE, but $33 \pm 4.18 \%$ in CQ+TACE. Furthermore, tumors of CQ+TACE were stained strongly positive with proapoptotic protein Bax and negative with antiapoptotic protein $\mathrm{Bcl}-2$ by immunohistochemistry; tumors of TACE alone were stained less strongly positive with Bax than CQ+TACE, and weakly strong with Bcl-2; in contrast, tumors of the control were negative with Bax and stongly positive with Bcl-2, which confirmed the higher level of apoptosis in CQ+TACE (Fig.2C and Fig.2D). There is no significant difference in necrosis between TACE alone and CQ+TACE, but much higher apoptosis in CQ+TACE, which suggests that CQ promotes the anticancer effect of TACE mainly by increasing 
apoptosis induced by chemotherapeutic agent. This is probably achieved by inhibiting autophagy although this has to be demonstrated subsequently.

\section{CQ inhibits autophagy induced by TACE}

Embolization in the TACE results in hypoxia [10, $33]$ and hypoxia can induce autophagy $[9,34,35]$. In our study, hypoxia resulted from TACE has been identified by expression of HIF-1 $\alpha$ (Fig. 1A). Then in order to demonstrate the hypothesis that increased apoptosis in CQ+TACE is the result of inhibited autophagy by $C Q$, autophagy activity in different groups was evaluated by immunohistochemical staining and Western blot with LC3 antibody. As shown in Fig. 3A and C, the expression of LC3 in the control and CQ alone was just in a basal level, but that in TACE was shown in a high level, while it was higher in CQ+TACE because CQ inhibits its degradation. In addition, the amount of p62, a selective sub- strate of autophagy and an autophagy-related marker, was measured. The p62 in the control and the CQ alone were strongly positive because it is normally accumulated without autophagy (Fig. 3B). Tissues in TACE alone, however, were stained weakly positive with p62. And within expectation, the amount of p62 increased again in CQ+TACE group because of the inhibited autophagy by CQ (Fig. 3B). Results above showed that autophagy was induced by TACE while CQ could effectively inhibit it. Autophagy induced by TACE enhanced the survivability of cancer cells, which mediated the resistance of cancer cells to chemotherapeutic agent. However, when the autophagy is inhibited by CQ, survivability of cancer cells decreased, and apoptosis caused by chemodrug increased. Then combination with CQ could eventually promote the efficacy of TACE.

A

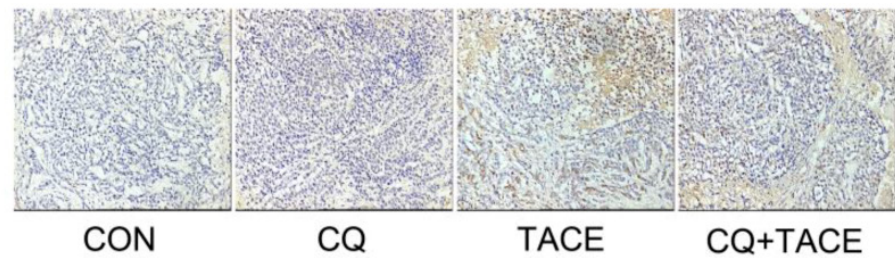

LC3

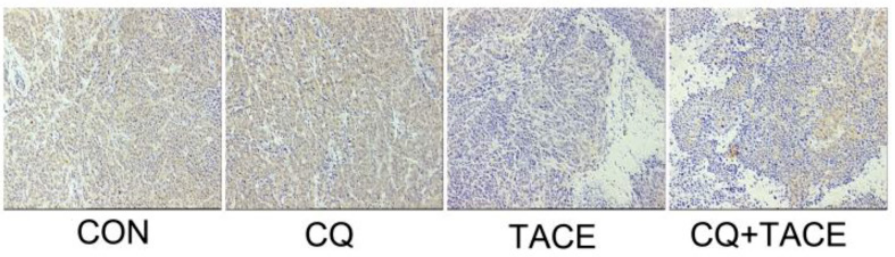

p62

B

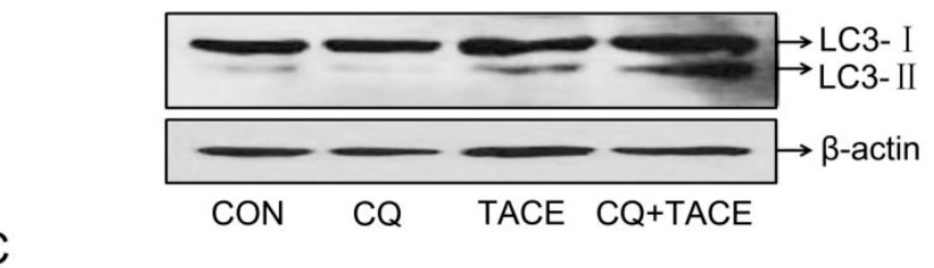

C

Fig 3. Expression of autophagy maker LC3 and p62 in immunohistochemical staining and immunoblotting assay . (A) Representative images of immunohistochemical staining with LC3 antibody in each group 14 days after the treatment. The LC3-positive cells were greatly increased in the groups receiving TACE, whereas CQ (CQ+TACE) diminished the expression of LC3. And there was a little positive cells shown in the control and CQ alone groups. (B) Immunohistochemistry against p62. Relative high expression of p62 was observed in the control and CQ groups. On the contrary, much less p62-positive cells could be found in the group of TACE as autophagy was activated by embolization. Then when CQ was added in CQ+TACE group, the level of p62 was increased again. Magnification, $\times 200$. (C ) The result of Western blot showed that the accumulation of LC3 was upregulated in TACE and CQ+TACE, but LC3II in CQ+TACE was higher than TACE. 

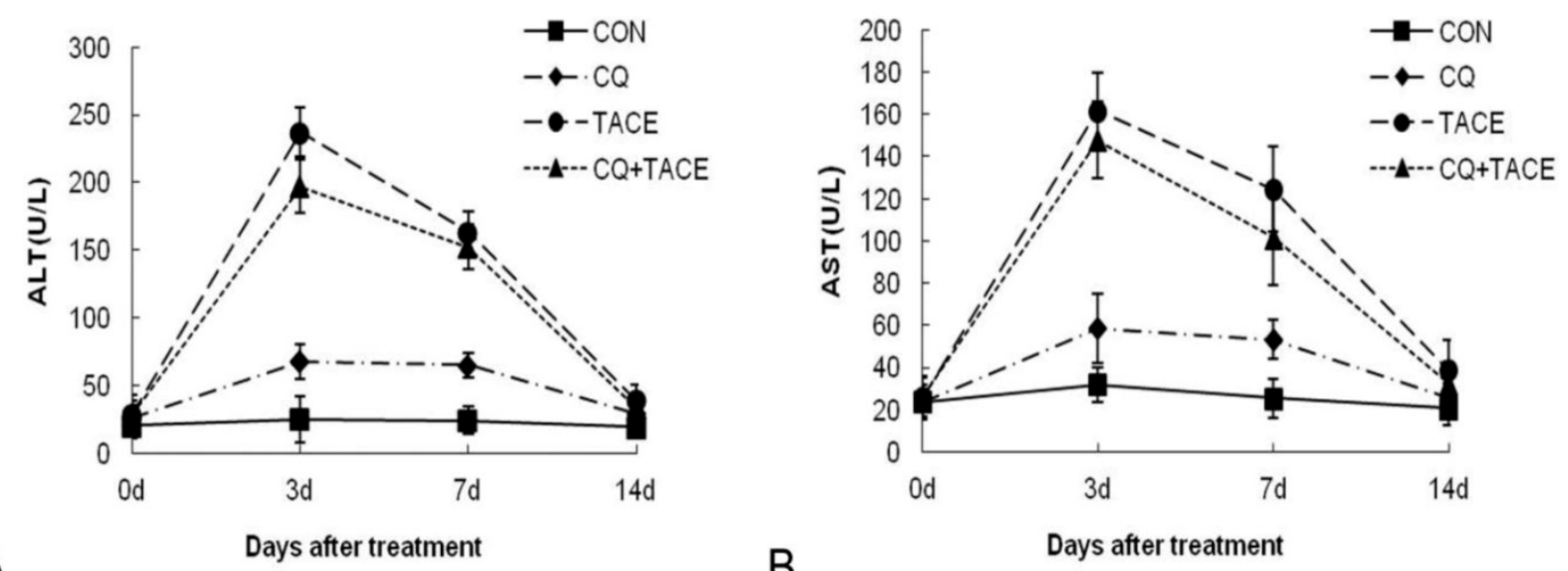

Fig 4. Assessment of the toxicity. Concentrations of ALT (A) and AST (B) in the blood increased transiently at 3 days after TACE whether CQ was combined or not. At 14 days after TACE or CQ+TACE, both of them returned to the levels before treatments in all groups. The values are shown as means \pm SD and the result showed no statistically significant difference between TACE or CQ+TACE group.

\section{CQ plus TACE is as safe as TACE}

Insults to nontumorous parenchyma are inevitably induced during TACE by embolic materials or anticancer drugs [36]. If additional CQ aggravates this damage severely, it can't be a promising adjuvant agent in TACE even though it could enhance tumor growth inhibition significantly. The safety of CQ+TACE was assessed in rabbit VX2 liver tumor model. A time-course study of alanine aminotransferase (ALT) plasma and aspartate aminotransferase (AST) concentrations before and after the treatments is shown in Fig. 4. At first, both ALT and AST levels of different groups were similar to each other. 3 days after the treatment, they increased transiently and significantly, but decreased at the $7 \mathrm{~d}$ time point. On the 14th day, ALT and AST concentrations in the group of CQ alone returned to the same normal level as the control, which indicated that CQ itself has no significant effect on liver function. But their level in TACE and CQ+TACE groups were significantly higher than the control 14 days after the treatments. However, both ALT and AST of CQ+TACE were not higher than TACE alone. Actually the level of ALT and AST in CQ+TACE group was more or less lower than that of TACE alone, but unfortunately this difference was not statistically significant. The goal of TACE is to promote tumor necrosis and control tumor growth while preserving as much functional liver tissue as possible [31]. Data above showed that liver injury caused by TACE may decrease when combined with CQ. Therefore, CQ may be used in clinical settings as an effective additive to TACE in the future to promote the efficacy of TACE.

\section{Discussion}

Hypoxia is one of the important factors that cause chemoresistance. In addition to the various mechanisms for chemoresistance induced by hypoxia, such as delivery and cellular uptake failure of drugs, loss of free radicals contributing to cytotoxicity, decrease of the proliferation rate of cancer cells, angiogenesis and so on, autophagy is the mechanism that also mediates chemoresistance in hypoxia but found afterwards. As one of the optimal treatments for advanced HCC, TACE shows its anticancer effect through causing cell death by embolization and chemotherapeutic agent. Embolization mainly causes necrosis while chemotherapeutic agent mainly causes apoptosis. However, because embolization results in a hypoxic microenvironment which contributes to chemoresistance, the effect of chemodrug is always poor. A large body of research has shown that TACE is not superior to TAE.

In order to promote the efficacy of TACE, especially to enhance the effect of chemodrug in TACE, we combined CQ, a well-known anti-malarial agent, that inhibits lysosomal acidification and blocks the terminal stage of autophagy [37], with TACE with the expectation that CQ would increase the chemosensitivity of cancer cells, because in our previous study, we found that autophagy mediates the chemoresistance of hepatocellular carcinoma cells in hypoxia. To mimic the clinical TACE treatment, rabbit VX2 liver tumor model was used and TACE was administered by punctuating through femoral artery. The combination of CQ and TACE showed predominant antitumor effect than the TACE alone as the tumor growth rate in the CQ+TACE was much lower than that in the 
TACE. This result is consistent with the previous reports that the combination of chemotherapeutic agent with autophagic inhibitor led to more pronounced tumor suppression in HCC xenografts [38, 39]. Meanwhile, the level of CD133 and EpCAM that is used to show the amount of remaining tumor-initiating cells was also examined by immunohistochemistry. The expression of EpCAM was not significantly different among the four groups, and the level of CD133 was higher in the groups of TACE and TACE+CQ, but there was no difference between these two groups (data not shown), suggesting that the combination of CQ and TACE may not affect the possibility of recurrence.

The mechanism underlying the significant anticancer effect of CQ+TACE was investigated subsequently. Necrosis was estimated at first as it is the main kind of cell death in TACE. Results showed that there is no difference in TACE and in CQ+TACE in necrosis. But analyzing all the results of H\&E staining, a little part of the tissue sections after TACE, whether with or without CQ, showed nearly thorough necrosis. This is precisely consistent with the results in clinical treatments where some of the patients were especially suitable for TACE that exceptionally good prognosis was attained. Knowing that there was no significant difference in necrosis, then apoptosis was analyzed. Data from TUNEL staining showed that there were much more apoptotic cells in CQ+TACE than TACE alone, and there was almost no apoptosis in the tissue section of CQ alone and the control, which demonstrated that CQ promoted the apoptosis induced by chemotherapeutic agent but not the effect of CQ itself. All of these results verified our hypothesis that CQ inhibits autophagy induced by hypoxia resulted from embolization in TACE, which in turn depresses the growth of cancer cells in hypoxia; cell death occurs consequently as they become more sensitive to apoptosis induced by chemotherapeutic agents. This is also supported by the observation in vitro in HeLa cells [40] as well as in vivo in murine model of head and neck squamous cell carcinoma [41]. Moreover, inhibition of active autophagy induces apoptosis and increases chemosensitivity in cholangio carcinoma [42].

Autophagy enables cancer cells to survive the harsh conditions of their microenvironment; it also enables them to sustain chemotherapy thus conferring resistance. High level of autophagy detected in cells exposed to anticancer agents signifies an adaptive response [37]. In our present study, we tested LC3 and measured the amount of a selective autophagy substrate. The results showed that autophagy was indeed induced by TACE. Previous studies report that it is unlikely that TACE alone would prolong survival, because TACE can evoke a variety of complications, usually arising from underlying causative factors including compromised liver function, main portal vein obstruction and so on[43]. One of the disadvantages of TACE was its subsequent damage of the liver function, which could be reduced by CQ slightly. The combination of CQ and TACE, therefore, can inhibit the tumor growth substantially without severe adverse effects on background liver function. This is convincing evidence for the promising application of $\mathrm{CQ}$ in clinical treatment in future. However, the mechanism is not clear. It may attribute to the specificity of CQ on cancer cells. Cancer cells can gain more autophagy activity than normal cells. Since CQ targets the cancer cells which show high level of autophagy, the chemotherapeutic agent is, therefore, easier to kill cancer cells so that less normal liver cells are attacked. Thus the liver function damage caused by TACE is reduced.

In conclusion, our study proposed a novel therapeutic strategy for liver cancer by demonstrating that combining CQ with TACE would improve the efficacy and safety of TACE. This strategy could be particularly beneficial to patients who are sensitive to CQ+TACE for improved survival.

\section{Acknowledgements}

Authors Lu Gao, Jian-rui Song and Jian-wei Zhang contributed equally to this article. This project was supported by National Natural Scien ce Foundation of China (grant numbers: 81030041, 31 171321, 81000970, 81101622, 81201584, 30921006); Key Basic Research Project of China (grant numbers: 2012 CBA01303, 2011CB966200, 2010CB945600); Special Fu nds for National key Sci-Tech Special Project of China (grant numbers: 2012ZX10002011, 2012ZX10002016); and Shanghai Science and Technology Committee (gr ant numbers: 12ZR1454200, 12ZR1439800, 11ZR14495 00, 10ZR1439600, 10411963100, 10ZR1439900, 1243190 0802).

\section{Conflicts of Interest}

The authors declare that there are no conflicts of interest.

\section{References}

1. Semela D, Heim M. Hepatocellular carcinoma. Ther Umsch. 2011; 68: 213-7.

2. Llovet JM, Di Bisceglie AM, Bruix J, Kramer BS, Lencioni R, Zhu AX, et al. Design and endpoints of clinical trials in hepatocellular carcinoma. J Natl Cancer Inst. 2008; 100: 698-711.

3. Parkin DM, Bray F, Ferlay J, Pisani P. Global cancer statistics, 2002. CA Cancer J Clin. 2005; 55: 74-108.

4. Takayasu K, Shima Y, Muramatsu Y, Moriyama N, Yamada T, Makuuchi $\mathrm{M}$, et al. Hepatocellular carcinoma: treatment with intraarterial iodized 
oil with and without chemotherapeutic agents. Radiology. 1987; 163: 345-51.

5. Kasugai H, Kojima J, Tatsuta M, Okuda S, Sasaki Y, Imaoka S, et al. Treatment of hepatocellular carcinoma by transcatheter arterial embolization combined with intraarterial infusion of a mixture of cisplatin and ethiodized oil. Gastroenterology. 1989; 97: 965-71.

6. Kamada K, Nakanishi T, Kitamoto M, Aikata H, Kawakami Y, Ito K, et al. Long-term prognosis of patients undergoing transcatheter arterial chemoembolization for unresectable hepatocellular carcinoma: comparison of cisplatin lipiodol suspension and doxorubicin hydrochloride emulsion. J Vasc Interv Radiol. 2001; 12: 847-54.

7. Choi GH, Kim DH, Kang CM, Kim KS, Choi JS, Lee WJ, et al. Is preoperative transarterial chemoembolization needed for a resectable hepatocellular carcinoma? World J Surg. 2007; 31: 2370-7.

8. Sugo H, Futagawa S, Beppu T, Fukasawa M, Kojima K. Role of preoperative transcatheter arterial chemoembolization for resectable hepatocellular carcinoma: relation between postoperative course and the pattern of tumor recurrence. World J Surg. 2003; 27: 1295-9.

9. Zhou WP, Lai EC, Li AJ, Fu SY, Zhou JP, Pan ZY, et al. A prospective, randomized, controlled trial of preoperative transarterial chemoembolization for resectable large hepatocellular carcinoma. Ann Surg. 2009; 249: 195-202.

10. Marelli L, Stigliano R, Triantos C, Senzolo M, Cholongitas E, Davies N, et al. Transarterial therapy for hepatocellular carcinoma: which technique is more effective? A systematic review of cohort and randomized studies. Cardiovasc Intervent Radiol. 2007; 30: 6-25.

11. Pleguezuelo M, Marelli L, Misseri M, Germani G, Calvaruso V, Xiruochakis E, et al. TACE versus TAE as therapy for hepatocellular carcinoma. Expert Rev Anticancer Ther. 2008; 8: 1623-41.

12. Williams KJ, Telfer BA, Xenaki D, Sheridan MR, Desbaillets I, Peters HJ, et al. Enhanced response to radiotherapy in tumours deficient in the function of hypoxia-inducible factor-1. Radiother Oncol. 2005; 75: 89-98.

13. Song J, Qu Z, Guo X, Zhao Q, Zhao X, Gao L, et al. Hypoxia-induced autophagy contributes to the chemoresistance of hepatocellular carcinoma cells. Autophagy. 2009; 5: 1131-44.

14. Carew JS, Medina EC, Esquivel JA, 2nd, Mahalingam D, Swords R, Kelly $\mathrm{K}$, et al. Autophagy inhibition enhances vorinostat-induced apoptosis via ubiquitinated protein accumulation. J Cell Mol Med. 2010; 14: 2448-59.

15. Poole B, Ohkuma S. Effect of weak bases on the intralysosomal pH in mouse peritoneal macrophages. J Cell Biol. 1981; 90: 665-9.

16. Glaumann H, Ahlberg J. Comparison of different autophagic vacuoles with regard to ultrastructure, enzymatic composition, and degradation capacity--formation of crinosomes. Exp Mol Pathol. 1987; 47: 346-62.

17. Tang H, Da L, Mao Y, Li Y, Li D, Xu Z, et al. Hepatitis B virus X protein sensitizes cells to starvation-induced autophagy via up-regulation of beclin 1 expression. Hepatology. 2009; 49: 60-71.

18. Shintani T, Klionsky DJ. Autophagy in health and disease: a double-edged sword. Science. 2004; 306: 990-5.

19. Klionsky DJ, Abeliovich H, Agostinis P, Agrawal DK, Aliev G, Askew DS, et al. Guidelines for the use and interpretation of assays for monitoring autophagy in higher eukaryotes. Autophagy. 2008; 4: 151-75.

20. Tanida I, Minematsu-Ikeguchi N, Ueno T, Kominami E. Lysosomal turnover, but not a cellular level, of endogenous LC3 is a marker for autophagy. Autophagy. 2005; 1: 84-91.

21. Pankiv S, Clausen TH, Lamark T, Brech A, Bruun JA, Outzen $H$, et al. p62/SQSTM1 binds directly to Atg8/LC3 to facilitate degradation of ubiquitinated protein aggregates by autophagy. J Biol Chem. 2007; 282: 24131-45.

22. Bjorkoy G, Lamark T, Brech A, Outzen H, Perander M, Overvatn A, et al. p62/SQSTM1 forms protein aggregates degraded by autophagy and has a protective effect on huntingtin-induced cell death. J Cell Biol. 2005; 171: 603-14.

23. Komatsu M, Kurokawa H, Waguri S, Taguchi K, Kobayashi A, Ichimura $\mathrm{Y}$, et al. The selective autophagy substrate p62 activates the stress responsive transcription factor Nrf2 through inactivation of Keap1. Nat Cell Biol. 2010; 12: 213-23.

24. Mathew R, Karp CM, Beaudoin B, Vuong N, Chen G, Chen HY, et al. Autophagy suppresses tumorigenesis through elimination of p62. Cell. 2009; 137: 1062-75.

25. Monick MM, Powers LS, Walters K, Lovan N, Zhang M, Gerke A, et al. Identification of an autophagy defect in smokers' alveolar macrophages. J Immunol. 2010; 185: 5425-35.

26. Begg AC, Stewart FA, Vens C. Strategies to improve radiotherapy with targeted drugs. Nat Rev Cancer. 2011; 11: 239-53.

27. Watanabe D, Ueo H, Inoue $H$, Matsuoka $H$, Honda $M$, Shinomiya $Y$, et al. Antitumor effects of intraarterial infusion of tumor necrosis factor/lipiodol emulsion on hepatic tumor in rabbits. Oncology. 1995; 52: 76-81.

28. Okada M, Kudo S, Miyazaki O, Saino T, Ekimoto H, Iguchi H, et al. Antitumoral efficacy and pharmacokinetic properties of pirarubicin upon hepatic intra-arterial injection in the rabbit VX 2 tumour model. $\mathrm{Br}$ J Cancer. 1995; 71: 518-24.

29. Higgins DF, Kimura K, Iwano M, Haase VH. Hypoxia-inducible factor signaling in the development of tissue fibrosis. Cell Cycle. 2008; 7: 1128-32.

30. Hiraga T, Kizaka-Kondoh S, Hirota K, Hiraoka M, Yoneda T. Hypoxia and hypoxia-inducible factor- 1 expression enhance osteolytic bone metastases of breast cancer. Cancer Res. 2007; 67: 4157-63.

31. Ramsey DE, Kernagis LY, Soulen MC, Geschwind JF. Chemoembolization of hepatocellular carcinoma. J Vasc Interv Radiol. 2002; 13: S211-21.

32. Yang ZF, Poon RT, To J, Ho DW, Fan ST. The potential role of hypoxia inducible factor 1alpha in tumor progression after hypoxia and chemotherapy in hepatocellular carcinoma. Cancer Res. 2004; 64: 5496-503.

33. Wu XZ, Xie GR, Chen D. Hypoxia and hepatocellular carcinoma: The therapeutic target for hepatocellular carcinoma. J Gastroenterol Hepatol. 2007; 22: 1178-82

34. Zakeri Z, Lockshin RA. Cell death: history and future. Advances in experimental medicine and biology. 2008; 615: 1-11.

35. Tracy K, Dibling BC, Spike BT, Knabb JR, Schumacker P, Macleod KF. BNIP3 is an RB/E2F target gene required for hypoxia-induced autophagy. Molecular and cellular biology. 2007; 27: 6229-42.

36. Kobayashi T, Teruya M. Preoperative transarterial chemoembolization for resectable large hepatocellular carcinoma. Ann Surg. 2010; 251: 386.

37. Levy JM, Thorburn A. Targeting autophagy during cancer therapy to improve clinical outcomes. Pharmacol Ther. 2011; 131(1):130-41.

38. Ding ZB, Hui B, Shi YH, Zhou J, Peng YF, Gu CY, et al. Autophagy activation in hepatocellular carcinoma contributes to the tolerance of oxaliplatin via reactive oxygen species modulation. Clin Cancer Res. 2011;17(19):6229-38

39. Guo XL, Li D, Hu F, Song JR, Zhang SS, Deng WJ, et al. Targeting autophagy potentiates chemotherapy-induced apoptosis and proliferation inhibition in hepatocarcinoma cells. Cancer Lett. 2012; 320: 171-9.

40. Liu XW, Su Y, Zhu H, Cao J, Ding WJ, Zhao YC, et al. HIF-1alpha-dependent autophagy protects HeLa cells from fenretinide (4-HPR)-induced apoptosis in hypoxia. Pharmacol Res. 2010; 62: 416-25.

41. Vigneswaran $\mathrm{N}, \mathrm{Wu} \mathrm{J}$, Song A, Annapragada A, Zacharias W. Hypoxia-induced autophagic response is associated with aggressive phenotype and elevated incidence of metastasis in orthotopic immunocompetent murine models of head and neck squamous cell carcinomas (HNSCC). Exp Mol Pathol. 2011; 90: 215-25.

42. Hou YJ, Dong LW, Tan YX, Yang GZ, Pan YF, Li Z, et al. Inhibition of active autophagy induces apoptosis and increases chemosensitivity in cholangiocarcinoma. Lab Invest. 2011; 91: 1146-57.

43. Shin SW. The current practice of transarterial chemoembolization for the treatment of hepatocellular carcinoma. Korean J Radiol. 2009; 10: 425-34. 